\title{
ELABORACIÓN Y ESPECIFICACIONES TÉCNICAS DEL PISCO PERUANO TERCERA ETAPA-PLANTA PROTOTIPO
}

\section{PREPARATION AND TECHNICAL SPECIFICATIONS OF THE PERUVIAN PISCO THIRD STAGE- PROTOTYPE PLANT}

\author{
Mario Ricardo De La Cruz Azabache ${ }^{1}$, Dionicio Adolfo Marcelo Astocóndor ${ }^{1}$ \\ RESUMEN
}

\begin{abstract}
El presente proyecto de investigación aplicada; tiene como objetivo definir las especificaciones técnicas de un producto de bandera como es el pisco peruano. La importancia de esta investigación, radica en el hecho que por ser la producción de pisco en el Perú mayormente artesanal y de poca tecnología, ésta no garantiza niveles estándar de calidad, composición y producción. Este trabajo contempla asimismo la obtención de un proceso estándar para la elaboración de aguardiente de uvas denominado Pisco, con pruebas de investigación a nivel de Reactor Prototipo, el desarrollo de producto a nivel comercial denominado Pisco UNI y la obtención de una Patente de Invención. Los resultados obtenidos a través de un trabajo multidisciplinario; con participación de investigadores y empresarios de instituciones como la Facultad de Ciencias Biológicas de la Universidad Nacional de San Marcos; UNMSM; el Centro de Innovación de la Vid; CITEvid y Bodega y Viñedos La Caravedo de Ica, fueron satisfactorios, obteniéndose un proceso estándar que garantiza la elaboración de un producto de calidad y protegido además con una Patente de Invención. Asimismo, el proyecto tuvo como objetivo suministrar herramientas de innovación tecnológica a los estudiantes de la Facultad de Ingeniería Química y Textil de la UNI; FIQT, a fin que puedan aplicarlos en el desarrollo de su carrera o en la creación de su propia empresa o fuente de trabajo, como una alternativa de desarrollo luego que egresen de la universidad
\end{abstract}

Palabras claves.- Investigación aplicada, Pisco Peruano. Aguardiente de uvas, Reactor prototipo, Multidisciplinario, Proceso estándar, Patente de Invención.

\begin{abstract}
The present project in Applied Research has the objective to define the technical specifications of Peruvian Pisco, a traditional drink and patrimony, symbol of Peruvian pride and nationality. The importance of this project lies in the search for a higher standard of quality, composition and production for pisco elaboration in Peru, which is mostly artisanal and low technology. This design also involves obtaining a standardized process for elaboration of the grape liqueur denominated Pisco, with research trials at Prototype Reactor level, the development of a commercial product named Pisco UNI and obtaining an Invention Patent. Through a multidisciplinary group with the participation of professionals from the Facultad de Ciencias Biológicas de la Universidad Nacional de San Marcos; UNMSM; the Centro de Innovación de la Vid; CITEvid and Bodega y Viñedos La Caravedo de Ica; the obtained results were satisfactory, the standardized process guaranties the elaboration of a product of high quality and protected by an Invention Patent. The project also aims to provide technological innovation tools to students from Facultad de Ingeniería Química y Textil de la
\end{abstract}

\footnotetext{
${ }^{1}$ MSc. Ingeniero investigador de la Facultad de Ingeniería Química y Textil de la Universidad Nacional de Ingeniería, ${ }^{2} \mathrm{MSc}$. Ingeniero investigador de la Facultad de Ingeniería Química y Textil de la Universidad Nacional de Ingeniería.
} 
UNI; FIQT, that they can apply in their career development or creating their own business or work source, as a development alternative after they graduate.

Key words.- Applied research, Peruvian Pisco, Grape liqueur, Prototype reactor, Multidisciplinary, Standard process, Patent of invention.

\section{INTRODUCCIÓN}

En un escenario de economía e información globalizada, las empresas de cualquier país, están sujetas a condiciones tales que se caracterizan por un requerimiento de alta competitividad y están expuestas a una variación continua de la tecnología. Lo anterior se agudiza debido al hecho que las empresas están obligadas a competir con empresas extranjeras establecidas fuera o dentro de su propio país.

El Pisco, además de ser una bebida tradicional del Perú desde tiempos de la Colonia española, constituye para el país lo que en el comercio internacional se conoce como denominación de origen. Según la definición establecida por la Organización Mundial de la Propiedad Intelectual (OMPI), se entiende por denominación de origen al nombre de un país, de una región o de un lugar determinado, que se utiliza para designar a un producto originario de ellos, cuyas cualidades y características se deben exclusiva y esencialmente al medio geográfico, incluidos los factores naturales (geografía, clima, materia prima, etc.) y los factores humanos (mano de obra, arte, ingenio, tradición, etc.).

Sin embargo, debido principalmente a factores estructurales de calidad, tecnología, capacidad de producción y comercialización, un gran porcentaje de empresas artesanales que elaboran pisco; no han logrado ampliamente la denominación de origen para su aguardiente de uva [1].

Lograr la denominación de origen en nuestro país; por parte de los productores; presenta dos dificultades: en primer lugar el costo elevado del trámite y en segundo lugar mantener la calidad del producto de acuerdo a normas específicas de producción. Asimismo es relevante en este contexto, la presencia de productos adulterados que atentan contra la fama de calidad del pisco peruano y la cantidad de productos de importación que ingresan al país incluso con denominación de Pisco [1].
La industria del pisco en el Perú; tiene una capacidad de producción de 2.3 millones de litros/año.

El objetivo del proyecto es el definir con precisión las especificaciones técnicas de un producto de bandera de calidad como es el pisco peruano y asimismo un proceso de producción que garantice la estandarización de esta calidad, apoyando principalmente al sector artesanal. En ese sentido; en el marco de los objetivos del proyecto; se firmó un convenio interinstitucional entre la UNI y el Centro e Innovación Tecnológica de la vid, CITEvid; lo que ha significado en un constante intercambio tecnológico entre ambas instituciones y que ha resultado en el establecimiento de parámetros técnicos de calidad para la elaboración del Pisco. CITEvid suministró en calidad de donación 300 kilos de uva Quebranta para pruebas en reactor de 45 litros [1].

El proyecto consideró la participación de la empresa privada a través de la Bodega y Viñedos La Caravedo de Ica, que suministró en calidad de donación 1 tonelada de uva Quebranta Orgánica con certificación internacional y luego en transacción comercial más de 6 toneladas para pruebas en Reactor de 600 litros.

Asimismo, como parte del trabajo de tipo multidisciplinario que propugna el proyecto; se contó con el apoyo de la Facultad de Biología de la Universidad Nacional Mayor de San Marcos.

El Pisco se procesa con uva en una razón de 6 kilos de uva por litro de pisco en promedio, y puede ser denominado: Pisco Puro si se elabora a partir de un solo tipo de uva, básicamente de la variedad "Quebranta". Pisco aromático, si se elabora a partir de una uva aromática por ejemplo uva "Italia". Pisco acholado, como producto de mezcla de varios tipos de uva o piscos. Las zonas productoras de uva en el Perú principalmente están en Lima, Ica, Arequipa, Moquegua y Tacna, siendo el Departamento de Ica el de mayor producción. 
En el Perú., la elaboración del Pisco está regulada mediante la Norma Técnica Peruana NTP 211.001INDECOPI. Sin embargo se debe precisar que en lo que se refiere a las especificaciones químicas así como en el proceso de fabricación, esta norma recoge en su mayor parte el carácter artesanal de la producción del Pisco en el Perú. Lo que explica el amplio rango entre los valores mínimos y máximos de los componentes del pisco que exige la norma y que posibilita que cualquier producto de dudosa calidad la cumpla.

La calidad del pisco pudo ser estandarizada a través de la definición de sus especificaciones técnicas y de un proceso de elaboración de aguardiente de uvas, y poniendo mucho énfasis en las etapas de pre-fermentación y fermentación del mosto o jugo de uva y su posterior destilación. A través de la investigación de estas etapas, se pudieron definir los parámetros de calidad y estandarizarlos. La estandarización incluyó además el diseño de los equipos principales tales como los reactores de pre fermentación y fermentación y el equipo de destilación batch [4].

Finalmente se procedió a la obtención de una Patente de Invención en INDECOPI para proteger el proceso.

A través del aprovechamiento del conocimiento adquirido en el campo de la biotecnología y utilizando los subproductos del proceso tales como la levadura y la cáscara de uva, se formularon las bases para desarrollar el Proyecto de Bioetanol a partir de materias primas nacionales, de primera y segunda generación, con resultados satisfactorios. Obteniéndose Bioetanol a partir de jugo de caña de azúcar y de papa y últimamente se viene investigando a partir de desechos celulósicos.

Asimismo se formula la creación de una empresa spin-off de Pisco-UNI como producto de la investigación e innovación tecnológica realizada en la FIQT, ayudando de esta manera a trasferir el conocimiento y la investigación científica al mundo empresarial (principalmente a la industria artesanal) buscando su aplicación directa en los procesos [4].

\section{ANTECEDENTES}

El proceso estandarizado y de calidad continua para la producción de Pisco-UNI fue el fruto de la investigación realizada en la FIQT de la UNI desde el año 2004 al 2008 bajo el nombre de "Elaboración y especificaciones técnicas del pisco peruano" en tres etapas diferenciadas de investigación. En 2004 se aprueba la etapa de investigación a Nivel Laboratorio, con pruebas a nivel reactor de 1 litro, obteniéndose resultados satisfactorios que permiten escalar la investigación a un nivel superior.

En 2005 se aprueba la segunda etapa del proyecto a Nivel Banco (Bench Scale) como pruebas a nivel de reactor-fermentador de 45 litros de capacidad, diseñado y construido para tal propósito. De igual manera considera realizar pruebas de destilación a ese nivel, utilizando un Destilador batch de 30 litros que se implementa en el Laboratorio 23-A de la FIQT. Paralelamente; como producto de las gestiones del equipo investigador en coordinación con las autoridades de la FIQT; en 2005 se firmó el Convenio Interinstitucional entre la Universidad Nacional de Ingeniería y el Centro de Innovación Tecnológica de la Uva, CITEvid, con el objetivo de apoyarse mutuamente en el desarrollo de la investigación aplicada relacionada al desarrollo de la actividad vitivinícola de los productores actuales y potenciales.

El convenio incluía un Primer Plan de Trabajo; el cual se complementó con la ejecución de la primera etapa de investigación del Proyecto aprobado por el Consejo de la FIQT. Como parte del convenio el CITEVID entrega 300 kilos de uva quebranta al proyecto y asimismo recibe como practicantes en su bodega de ICA a 4 estudiantes miembros del proyecto de investigación.

A partir de los resultados positivos de la Escala Banco, se determina un proceso preliminar para la elaboración del aguardiente de uva, cuya calidad es evaluada en las instalaciones del CITEvid con catador oficial.

Se configura entonces una tercera etapa de investigación a Escala de Reactor-Fermentador de 600 litros, con el objetivo de definir un proceso definitivo de elaboración de aguardiente de uva denominado Pisco con estándares de calidad y producción definidos.

Otro de los objetivos era el diseñar y construir los primeros equipos prototipos para la elaboración de Pisco y a la vez adaptar equipos disponibles en el 
Laboratorio 23-A de la FIQT. En ese sentido, se diseñó, construyó e implementó un Reactor Fermentador de 600 litros de acero grado sanitario. Ver Fig. 1 y se implementó un destilador batch de 30 litros, provisto de condensador y enfriador, con control de temperatura en el hervidor y con registro continuo de la energía suministrada, Ver Fig. 2.

El objetivo para definir el proceso de elaboración de aguardiente de uvas denominado Pisco, contemplaba desde la etapa del suministro de la materia prima; uva quebranta en racimos; hasta la elaboración del producto final; pisco envasado en botellas y debidamente etiquetado [4].

Para el suministro de la materia prima se hizo un convenio tecno comercial con la Bodega y Viñedos La Caravedo de Ica; para el suministro de uva quebranta orgánica, en una cantidad de aproximada de 1 tonelada de uva quebranta por lote de reactor-fermentador.

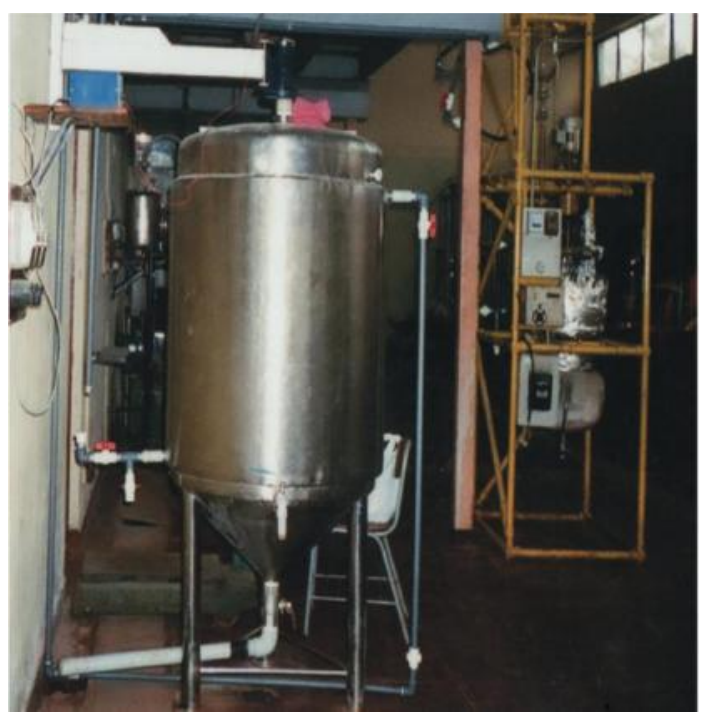

Fig. 1 Reactor-Fermentador prototipo de 600 litros.

Otro de los objetivos consistía en la optimización de cada una de las etapas del proceso; como por ejemplo la utilización de los subproductos tales como el orujo, cáscaras, pepas, anhídrido carbónico y la levadura Saccharomyce cerevisiae que se obtenía masivamente a modo de "borra" en la etapa de fermentación.

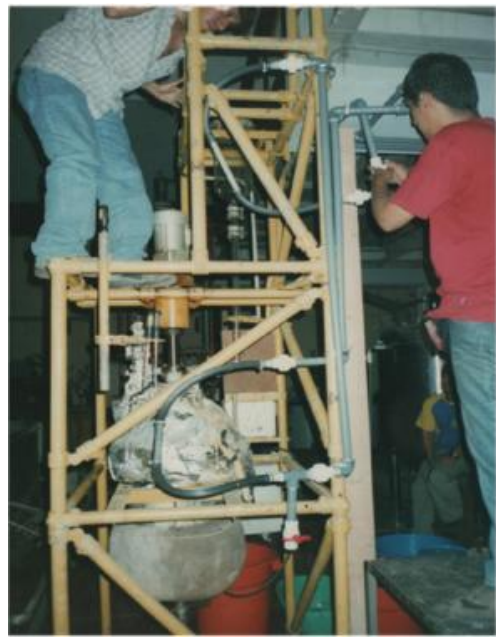

Fig. 2 Destilador batch con condensador y enfriador.

El proyecto consideró la participación masiva de alumnos investigadores de la FIQT; en un trabajo comprometido con los investigadores principales; así como del apoyo constante de un equipo multidisciplinario, principalmente de la Facultad de Ciencias Biológicas de la Universidad Nacional Mayor de San Marcos.

\section{PROCEDIMIENTO EXPERIMENTAL}

El procedimiento experimental cubrió los siguientes aspectos [4]:

- Construir base de datos relevante sobre el pisco peruano.

- Definir las especificaciones técnicas del pisco.

- Definir las variables de control del proceso para la elaboración de pisco.

- Diseño y construcción de los equipos prototipos para desgranar y prensar uva con fines de elaborar pisco.

- Diseño y construcción del equipo prototipo para fermentar mosto de uva.

- Diseño y construcción del equipo de destilación de mosto fermentado de uva.

- Definición y desarrollo del proceso para elaboración de aguardiente de uva denominado Pisco y

- Obtención de Patente de Invención.

El método consistió en realizar varias corridas experimentales, centradas en las etapas de pre- 
fermentación y fermentación del mosto o jugo de uva en el Reactor-Fermentador y luego la destilación del mosto fermentado en el destilador batch. Sin embargo, con el fin de precisar en detalle el proceso de elaboración se controló todas las variables relevantes en cada una de sus etapas. Esta información sirvió para desarrollar los balances de materia y energía y costos de producción en todo el proceso. Se inicia el proceso "despalillando" los racimos de uva, esto es; separando el raspón o escobajo de las vallas o granos de uva, las que se prensan con una presión controlada en un rango de 0.3-0.6 kg/cm2; suficiente para separar el mosto de la uva del orujo sin maltratar la cáscara y evitando daño en las pepas de la uva. Ver Fig. 3.

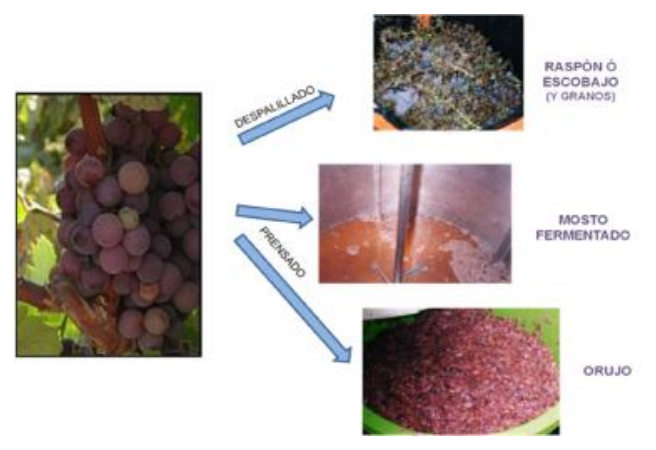

Fig. 3 Productos del despalillado y prensado de la uva.

El mosto obtenido se envía al reactor fermentador de 600 litros; dándose inicio a la fermentación debido a las levaduras que vienen con la uva, Ver Fig. 4, mientras que el raspón o escobajo se retira como subproducto del proceso para otros usos industriales y/o comerciales.

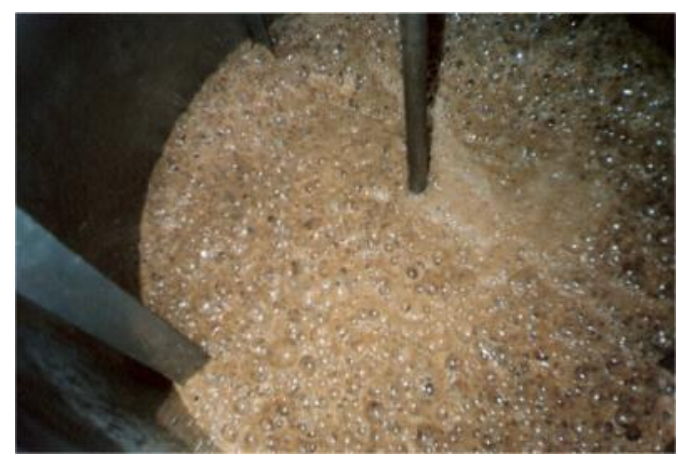

Fig. 4 Mosto o jugo de uva en proceso de fermentación
Se toma la temperatura, el PH y la variación de densidad del mosto que está relacionada con la variación de la generación del alcohol etílico presente en el mosto. Se mide la variación de nivel del mosto en el reactor fermentador. $\mathrm{La}$ temperatura se mantiene en el rango de $28-32{ }^{\circ} \mathrm{C}$, rango óptimo según los resultados obtenidos en la Escala Banco [2 y 3].

Los sólidos obtenidos en la etapa de prensado, en forma de una pasta denominada "orujo" de naturaleza seudo plástica por la presencia de fibras, cáscaras e hidrocoloides [2 y 3]se envía a un Reactor Pre-fermentador provisto de un agitador de velocidad lenta y con doble función: facilitar en primer lugar; la acción de las levaduras en los azúcares impregnados en el orujo y adheridos a la cáscara de la uva; dispersándolas en esta acción; y en segundo lugar separar las pepas, las cuales, se asientan en el fondo del tanque.

Al tercer día en el pre-fermentador, la cáscara se separa nítidamente, sobrenadando sobre el mosto pre fermentado. Por efecto de la agitación controlada; las pepas se sedimentan en su mayor parte en el fondo del Reactor pre-fermentador de donde son retiradas. De las pepas se pueden extraer aceite con características antioxidantes y otros subproductos tanto con métodos de extracción con solventes o mediante la extracción a presión en frío

El sólido sobrenadante en el Reactor-Pre fermentador se centrifuga con el fin de separar eficientemente la cáscara del mosto prefermentado, que junto al que se obtiene por decantación, se envía al Reactor-Fermentador con el fin que complete su fermentación por un total de 7-11 días

El Reactor Fermentador, está provisto de un sistema de agitación que tiene como función principal mantener uniforme la concentración de los azúcares y de las levaduras así como el grado de suspensión de estas últimas en cada punto del fermentador [7 y 8]. Esto es de suma importancia, dado que conforme avanza el proceso de fermentación, el contenido de azúcares disminuye, reduciéndose sustancialmente la densidad del líquido, lo que provoca a su vez la sedimentación de la levadura y 
por tanto la velocidad de fermentación, provocando "stress" en la levadura y en consecuencia detrimento de la calidad del mosto.

El nivel de agitación se mantuvo de tal manera que no se perturbaba la acción de la levadura trabajándose con un máximo de agitación que no superase un valor de Numero de Reynolds de mezclado (Nre) de 8,000 -14,000.

Asimismo, al fermentador se le implementó un sistema de enfriamiento-calentamiento, que aseguraba un nivel de temperatura en el rango de $28^{\circ} \mathrm{C}-32{ }^{\circ} \mathrm{C}$. Cuando la temperatura está por debajo de $\operatorname{los} 25^{\circ} \mathrm{C}$ es difícil que se active eficazmente la levadura y si está por encima de los $35^{\circ} \mathrm{C}$ puede causarle daño.

La baja temperatura en el reactor dependía de la temperatura del medio ambiente y la alta temperatura se presenta cuando se lleva a cabo la fermentación tumultuosa donde la temperatura en el Reactor Fermentador podía elevarse por encima de $45^{\circ} \mathrm{C}$.

El sistema de enfriamiento- calentamiento mantiene la temperatura del Reactor Fermentador en el rango $28^{\circ} \mathrm{C}-32^{\circ} \mathrm{C}$.

Los cálculos de rendimiento teórico en la fermentación, a través de la producción de alcohol etílico, se obtienen haciendo uso de las reacciones estequiométricas globales (1) y (2).

Hidrólisis de la sacarosa.-

$\mathrm{C}_{12} \mathrm{H}_{22} \mathrm{O}_{11}+\mathrm{H}_{2} \mathrm{O} \rightarrow \mathrm{C}_{6} \mathrm{H}_{12} \mathrm{O}_{6}+\mathrm{C}_{6} \mathrm{H}_{12} \mathrm{O}_{6}$

Sacarosa Agua Glucosa Fructuosa

Degradación de la glucosa y/o fructuosa.-

$$
\begin{gathered}
2 \mathrm{C}_{6} \mathrm{H}_{12} \mathrm{O}_{6} \stackrel{\text { Enzima }}{\longrightarrow} 4 \mathrm{C}_{2} \mathrm{H}_{5} \mathrm{OH}+4 \mathrm{CO}_{2}+\text { Calor } \\
\text { Glucosa } \\
\text { Etanol Gas Carbónico }
\end{gathered}
$$

(Fructuosa)

La glucosa y la fructuosa son isómeras. Cada una de ellas es sintetizada por acción de la levadura Saccharomyces cerivisiae para formar dos (2) moléculas de etanol y dos (2) moléculas de dióxido de carbono, así como otros productos secundarios en una mínima proporción y con un gran desprendimiento de calor, $8,5 \mathrm{Kcal} / \mathrm{mol} \mathrm{de}$ etanol formado.
Tanto en el pre fermentador como en el fermentador; como resultado de la fermentación de los azúcares, se forma anhídrido carbónico que se retira en forma controlada. Sin embargo; se mantiene a través del diseño del ReactorFermentador; un "colchón" de anhídrido carbónico en el espacio comprendido entre la superficie del mosto en fermentación y la parte superior del reactor; con el objetivo de lograr un proceso puramente anaeróbico que optimice la generación de etanol.

Al finalizar la fermentación se detiene la agitación y la levadura se sedimenta. Luego el mosto fermentado se clarifica obteniéndose un vino de mosto de uva fermentado y un lodo de levadura sedimentado, denominado "borra". Ver Fig. 5.

La "borra" contiene mosto fermentado en una proporción de 8-10\% muy difícil de filtrar. El equipo de investigación determinó el equipo de filtración apropiado para la recuperación del mosto [5 y 6]. Los sólidos obtenidos del filtro es levadura húmeda, que se recupera para su aprovechamiento posterior, generalmente para utilizarla en otros procesos de fermentación.

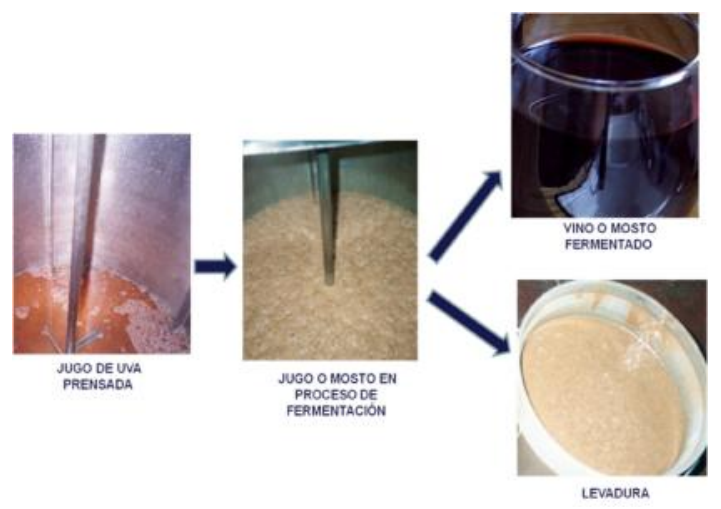

Fig. 5 Productos de la fermentación del mosto de uva.

Todo el mosto de uva fermentado se colecta en un tanque de retén, de donde se alimenta al destilador batch por la parte de hervidor de acero inoxidable Ver Fig. 6.

El mosto se calienta hasta la temperatura de evaporación de los alcoholes y compuestos aromáticos que configuran el aguardiente denominado Pisco. El flujo de calor se alimenta con flujo constante a través del sistema de 
calentamiento y controlando la temperatura del vapor.

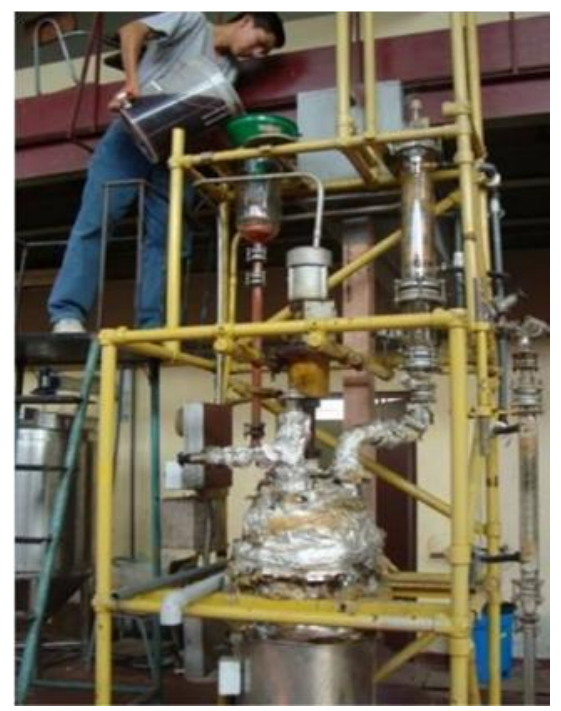

Fig. 6 Alimentación de mosto fermentado a destilador batch.

En la destilación del mosto fermentado, se separa un primer corte denominado "cabeza" que se caracteriza por su alto grado alcohólico por encima de $75^{\circ} \mathrm{GL}$ y contiene compuestos indeseados para el aguardiente. Se obtiene cuando la temperatura en fase vapor toma valores máximos de $88.5^{\circ} \mathrm{C}$. Este corte representa el $2.3-2.5 \%$ de todo el aguardiente destilado y se retira del proceso para otros usos industriales y/o comerciales.

Luego de destilado y separado el corte denominado "cabeza", se prosigue destilando en forma continua hasta que el grado alcohólico del aguardiente acumulado sea de $38-46{ }^{\circ} \mathrm{GL}$, denominándose a esta porción del aguardiente destilado "cuerpo", el cual se obtiene en el rango de temperatura de vapor máxima de $99{ }^{\circ} \mathrm{C}$. El "cuerpo" se almacena en tanques de maduración durante un periodo de 3 a 6 meses.

El residuo del mosto fermentado que queda luego de destilarse el aguardiente de uva pisquera se denomina "cola" y se retira para otros usos industriales y/o comerciales.

El proceso presentado para la obtención óptima de aguardiente de uva denominado Pisco considera un sistema de recirculación del agua de enfriamiento utilizado en el enfriador y que luego se lleva a un tanque provisto de un elemento enfriador de donde el agua retorna permanentemente al enfriador.

De este sistema de enfriamiento también se recircula agua fría al Reactor-Fermentador, en los casos que se requiere enfriar, la cual retorna, optimizando en todos los casos el consumo de un elemento valioso como es el agua.

\section{RESULTADOS Y DISCUSIÓN}

\section{Fermentación y pre fermentación-rendimientos}

En la Fig. 7 se muestra la variación de la densidad del mosto en proceso de fermentación, que indica la transformación de los azúcares en etanol, y en Tabla 1, la data recolectada en cada prueba de fermentación en el Reactor - Fermentador.

Nótese que en el primer día no se nota mayor cambio en la densidad del mosto.

Es la etapa de crecimiento de la levadura o fase de inducción, luego dentro de los 4 siguientes días hay una caída dramática de la densidad, fase de la fermentación tumultuosa con gran desprendimiento de calor y gran generación de etanol y anhídrido carbónico, como producto de la reacción enzimática de los azúcares presentes.

Finalmente se presenta la fase de fermentación lenta con una reducida variación de la densidad. La fase de inducción se caracteriza por ser aeróbica y donde se lleva a cabo el crecimiento de la levadura.

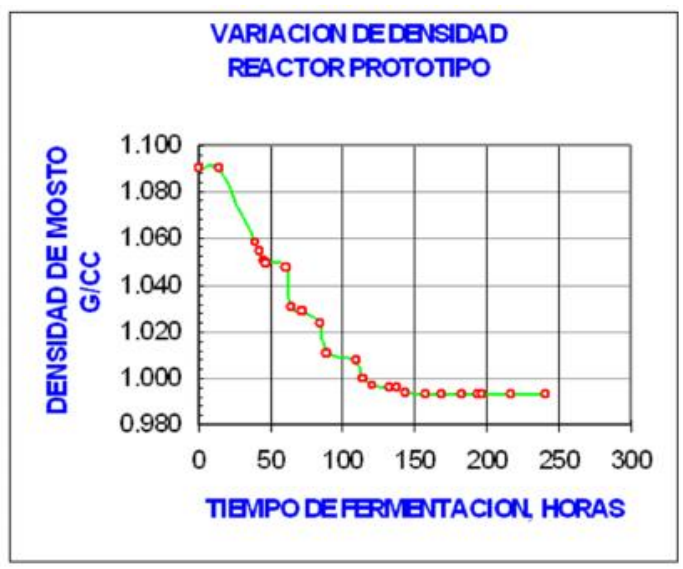

Fig. 7 Variación de la densidad en reactor fermentador. 
La fermentación tumultuosa es un proceso anaeróbico y se lleva a cabo en ausencia de oxígeno, asimismo sucede en la fase de fermentación lenta.

Tabla 1. Data recolectada en el control de cada corrida en el reactor - fermentador 600 litros.

\begin{tabular}{|c|c|c|c|c|c|c|c|}
\hline \multirow{2}{*}{$\begin{array}{l}\text { Control } \\
\text { № }\end{array}$} & \multirow[b]{2}{*}{ Fecha } & \multirow[b]{2}{*}{ Hora } & \multicolumn{4}{|c|}{ MOSTO } & \multirow{2}{*}{$\begin{array}{l}\text { Agitador } \\
\text { RPM }\end{array}$} \\
\hline & & & 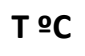 & oBé & $\rho, \mathrm{gr} / \mathrm{ml}$ & $\mathrm{pH}$ & \\
\hline 1 & 16.03 .07 & $12: 00$ & 28,0 & 11,0 & 1,085 & 3,58 & 34 \\
\hline 2 & 16.03 .07 & 20:08 & 28,0 & 11,0 & 1,084 & 3,64 & 34 \\
\hline 3 & $17,03,07$ & 08:30 & 28,0 & 10,0 & 1,070 & 3,57 & 34 \\
\hline 4 & $17,03,07$ & $15: 18$ & 29,0 & 9,0 & 1,065 & 3,54 & 30 \\
\hline 5 & $18,03,07$ & 09:00 & 27,0 & 7,3 & 1,050 & 3,50 & 30 \\
\hline 6 & $18,03,07$ & $18: 00$ & 28,0 & 6,0 & 1,043 & 3,53 & 30 \\
\hline 7 & $19,03,07$ & $08: 30$ & 30,0 & 5,0 & 1,034 & 3,53 & 30 \\
\hline 8 & $19,03,07$ & $17: 15$ & 29,0 & 4,2 & 1,029 & 3,59 & 30 \\
\hline 9 & $19,03,07$ & $20: 40$ & 29,0 & 4,2 & 1,029 & 3,64 & 30 \\
\hline 10 & 20.03 .07 & $08: 45$ & 29,0 & 3,9 & 1,023 & 3,64 & 30 \\
\hline 11 & 20.03 .07 & $14: 45$ & 29,0 & 3,5 & 1,022 & 3,70 & 30 \\
\hline 12 & 20.03 .07 & $20: 00$ & 25,0 & 3,5 & 1,022 & 3,74 & 30 \\
\hline 13 & 21.03 .07 & $08: 10$ & 26,5 & 3,0 & 1,020 & 3,72 & 30 \\
\hline 14 & 21.03 .07 & $14: 45$ & 28,5 & 2,5 & 1,015 & 3,73 & 30 \\
\hline 15 & 21.03 .07 & $20: 28$ & 27,0 & 2,0 & 1,013 & 3,81 & 30 \\
\hline 16 & 22.03 .07 & 08:45 & 27,0 & 1,8 & 1,011 & 3,76 & 30 \\
\hline 17 & 22.03 .07 & $20: 00$ & 29,0 & 1,5 & 1,008 & 3,77 & 30 \\
\hline 18 & 23.03 .07 & $08: 20$ & 28,0 & 1,3 & 1,007 & 3,74 & 30 \\
\hline 19 & 23.03 .07 & $14: 40$ & 28,0 & 1,0 & 1,005 & 3,78 & 30 \\
\hline 20 & 23.03 .07 & $18: 30$ & 29,0 & 1,0 & 1,005 & 3,82 & 30 \\
\hline 21 & 24.03 .07 & $11: 00$ & 28,0 & 0,8 & 1,003 & 3,89 & 30 \\
\hline 22 & 24.03 .07 & $17: 45$ & 27,5 & 0,7 & 1,002 & 3,90 & 30 \\
\hline 23 & 25.03 .07 & 09:00 & 28,0 & 0,3 & 1,001 & 3,91 & 30 \\
\hline 24 & 26.03 .07 & $08: 20$ & 29,0 & 0,3 & 0,998 & 3,92 & 30 \\
\hline 25 & 26.03 .07 & $15: 36$ & 28,0 & $<0,0$ & 0,996 & 3,90 & 30 \\
\hline 26 & 27.03 .07 & 08:20 & 29,0 & $<0,0$ & 0,995 & 3,91 & 30 \\
\hline
\end{tabular}

Por tanto, en el diseño del Reactor-Fermentador; se debió contemplar esta variación de las condiciones de la reacción durante todo el proceso de fermentación, con el fin de garantizar niveles altos de rendimiento y calidad del mosto fermentado.

La introducción de una etapa innovadora de prefermentación en el proceso desarrollado por el equipo investigador, se fundamenta en dos objetivos claros: recuperación de azúcares fermentables y de los compuestos aromáticos y de sabor que usualmente se desechan con el "orujo" en la industria del Pisco, dado que es muy difícil extraerlos por medios mecánicos, por la naturaleza seudo plástica de este material y por el riesgo de dañar el mosto incorporando materiales indeseables de las cáscaras y pepas. En la pre fermentación del "orujo" se recuperan selectivamente estos materiales mediante 
mecanismos enzimáticos con dos resultados positivos: aumento del rendimiento y mejora organoléptica del mosto y por tanto del aguardiente.

Los resultados obtenidos en la escala de Reactor Prototipo de 600 litros, son similares a los obtenidos en la escala banco de reactor de 45 litros. Otro resultado importante de la investigación y que era de interés de la Bodega y Viñedos La Caravedo de Ica, era que con el proceso obtenido podía elaborarse el aguardiente; con excelentes resultados; en cualquier momento del año, realizándose pruebas de comprobación en meses donde usualmente ya no se elabora este aguardiente. Ver Fig. 8 y Tabla 2.

Tabla 2. Resultados obtenidos en la elaboración de aguardiente de uva denominado Pisco a escala de Reactor - Fermentador de 600 litros.

\begin{tabular}{|c|c|c|c|c|}
\hline & M1-07 & M2-07 & M3-07 & M4-07 \\
\hline Fecha de recepción & 02.03 .07 & 11.03 .07 & 23.03 .07 & 02.04 .07 \\
\hline Lugar de recepción & UNI & UNI & UNI & UNI \\
\hline Cajas de 10 kilos aprox. & 75 & 71 & 77 & 110 \\
\hline Peso Neto de Uva, kg & 805,6 & 741,5 & 811,1 & 1187,3 \\
\hline Peso de granos, kg & 756,4 & 697,6 & 752 & 1113,8 \\
\hline Peso de palillo, kg & 29,6 & 21,7 & 23,3 & 34,4 \\
\hline Peso de pasas, kg & 19,6 & 22,2 & 35,8 & 39,1 \\
\hline$\%$ Grano en uva & 93,9 & 94,1 & 92,7 & 93,8 \\
\hline$\%$ Palillo en uva & 3,7 & 2,9 & 2,9 & 2,9 \\
\hline \% Pasa en Uva & 2,4 & 3,0 & 4,4 & 3,3 \\
\hline Uva efectiva a proceso, $\mathrm{kg}$ & 779,8 & 719,2 & 775,4 & 1148,1 \\
\hline \multicolumn{5}{|l|}{ Densidad, kg/litro } \\
\hline - Mosto Virgen & 1,093 & 1,085 & 1,093 & 1,102 \\
\hline - Mosto fermentado & 0,995 & 0,995 & 0,995 & 1,004 \\
\hline Mosto fermentado, litros & 550,5 & 496,4 & 536,3 & 800,0 \\
\hline Periodo fermentación, días & 10,8 & 10,8 & 6,9 & 18,0 \\
\hline Mosto destilado, litros & 529,0 & 484,7 & 496,5 & 778,0 \\
\hline Destilado, litros @ 46 oGL & 166,6 & 147,0 & 158,9 & 237,3 \\
\hline Destilado/Mosto destil. \% & 31,5 & 30,3 & 32,0 & 30,5 \\
\hline Kg uva neta / litro destilado & 4,8 & 5,0 & 5,1 & 5,0 \\
\hline Kg uva efectiva / litro destilado & 4,7 & 4,9 & 4,9 & 4,8 \\
\hline
\end{tabular}




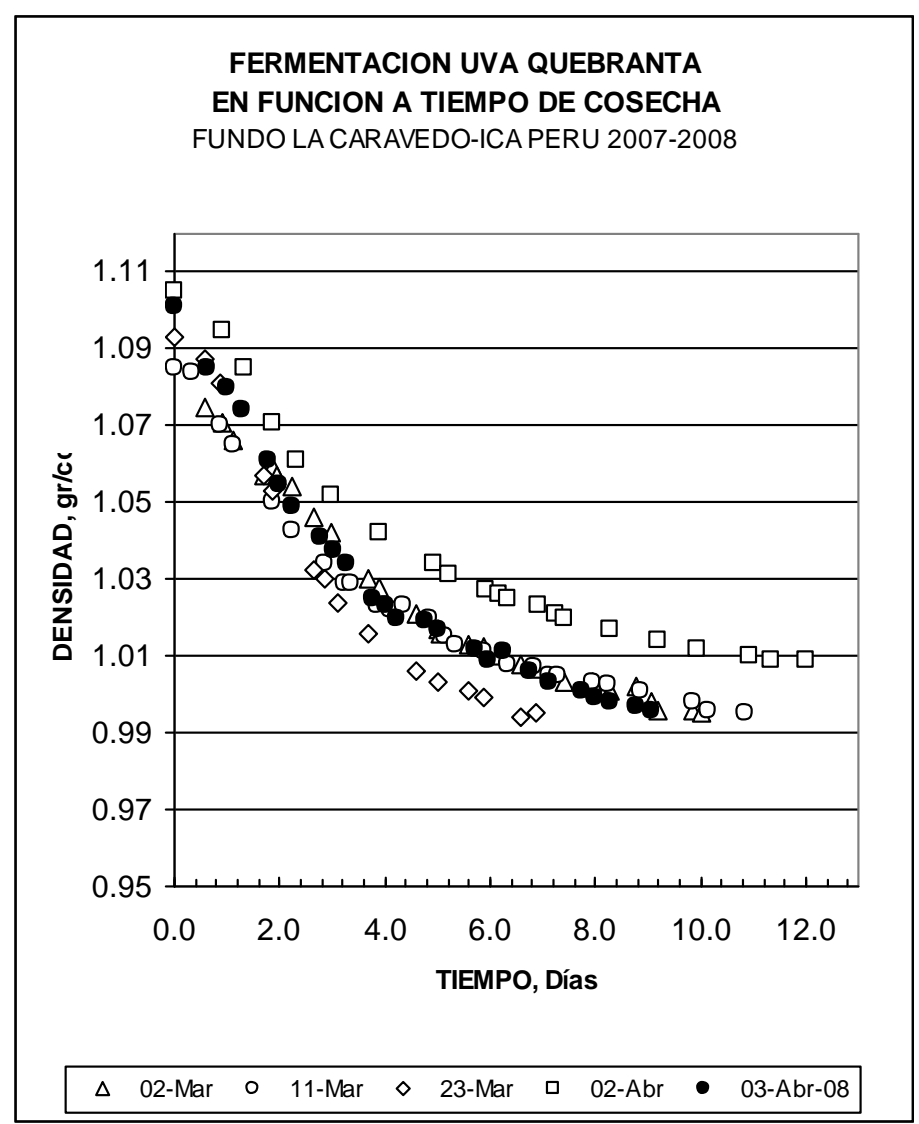

Fig. 8 Variación de la densidad en Reactor Fermentador de 600 litros, en épocas de no vendimia.

Asimismo se incorpora una etapa de recuperación de mosto fermentado adicional a partir de la "borra", o lodo de levadura Saccharomyces cerivisiae, utilizando un sistema de filtración adecuado a las especiales características de este lodo, obteniéndose mosto fermentado adicional, con un incremento del rendimiento del proceso [5 y 6].

Como resultado de las innovaciones en las etapas de fermentación, pre fermentación y tratamiento de la "borra", se aumenta sustancialmente el rendimiento de transformación de la uva a mosto fermentado y por tanto a pisco.

El consumo de uva se reduce hasta alrededor de 5 kilos de uva por litro de aguardiente producido, muy por debajo del estándar de la industria.

\section{Destilación del mosto fermentado}

En cada lote del reactor fermentador se generaba alrededor de 500 litros de mosto fermentado, el cual se procesaba en un destilador batch de 30 litros, generando aprox. 17 pruebas de destilación por lote de fermentación. Para la determinación precisa de la "cabeza", "cuerpo" y "cola", se colectó "cortes" de 500 cc, 1000 cc y 1500 cc. de destilado. Registrándose temperaturas de destilación, densidad, contenido alcohólico de cada corte y del acumulado y el consumo de energía, como se puede apreciar en las Tablas 3 y 4 . La Fig. 9 muestra la variación del contenido alcohólico del corte en función del porcentaje de corte de diversas pruebas de destilación.

Asimismo fue necesario definir una variable de control para precisar el \% de destilado 
correspondiente a la "cabeza" y que debería rechazarse.

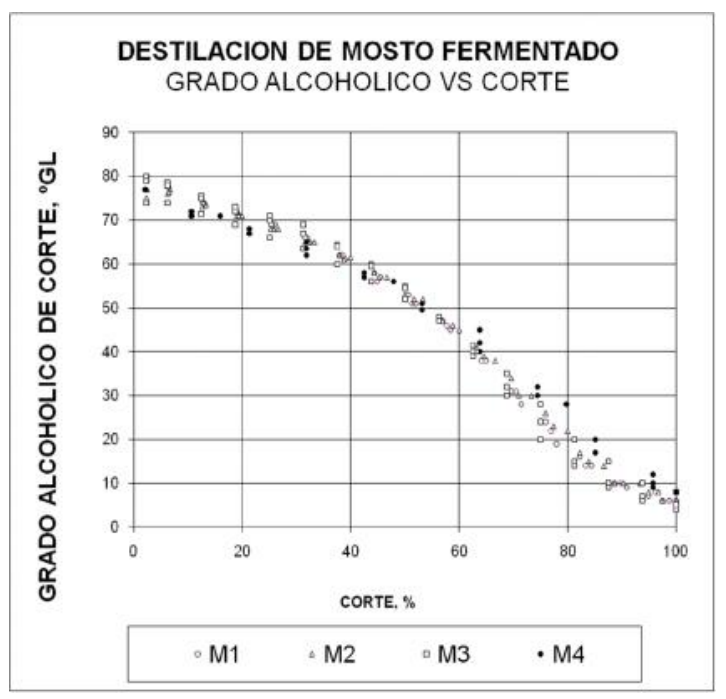

Fig. 9 Variación del grado alcohólico en función al \% de corte.
Esta fue la temperatura del vapor. La Fig. 10 muestra el perfil de temperatura de todo el proceso de destilación y donde se fija el rango de temperatura correspondiente a la "cabeza" del destilado.

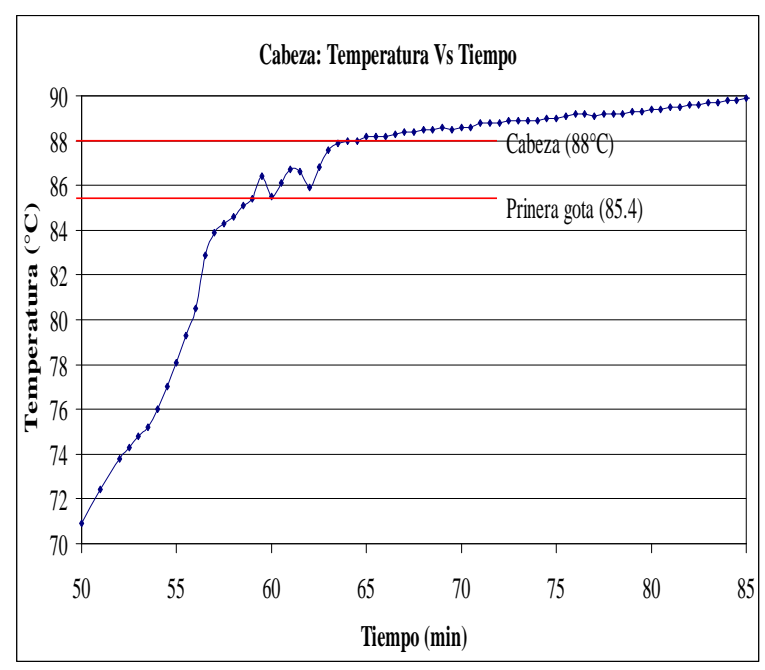

Fig. 10 Perfil de temperatura en la destilación del mosto.

Tabla 3. Data recolectada en el control de cada corrida en el destilador Batch de 30litros.

\section{FORMATO DE CONTROL DE DESTILACIÓN DEL VINO BASE}

\begin{tabular}{|c|c|c|c|c|c|c|c|c|c|c|}
\hline \multicolumn{3}{|c|}{$\begin{array}{l}\text { Fecha:25.04.06 } \\
\text { Primera Gota: 09:55 AM }\end{array}$} & \multicolumn{4}{|c|}{$\begin{array}{l}\text { Hora de inicio: } 08: 15 \text { AM } \\
\text { T. Vapor }=86,3 \stackrel{\circ}{\circ} \mathrm{C}\end{array}$} & \multicolumn{4}{|c|}{$\begin{array}{l}\text { Volumen de Mosto: } 25 \mathrm{~L} \\
\text { Marcador de Energía: 1230,9 Kw }\end{array}$} \\
\hline $\begin{array}{c}\text { № } \\
\text { Corte }\end{array}$ & $\begin{array}{c}\text { Volumen } \\
\mathrm{ml}\end{array}$ & $\begin{array}{c}\mathbf{O G} \\
\text { muestra }\end{array}$ & $\begin{array}{l}\rho, \mathbf{g r} / \mathrm{ml} \\
\text { muestra }\end{array}$ & $\begin{array}{c}\text { To } \mathrm{C} \\
\text { muestra }\end{array}$ & $\begin{array}{c}\text { o GL } \\
\text { mezcla }\end{array}$ & $\begin{array}{c}\rho, \mathrm{gr} / \mathrm{ml} \\
\mathrm{mezcla}\end{array}$ & $\begin{array}{c}\text { To C } \\
\text { mezcla }\end{array}$ & Tiempo & $\begin{array}{l}\text { To C } \\
\text { Vapor }\end{array}$ & $\begin{array}{c}\text { Energía } \\
\mathrm{Kw}\end{array}$ \\
\hline Cabeza & 188 & 70,5 & $<0,9$ & 25,0 & 70,5 & $<0,9$ & 25,0 & $10: 00$ & 89,1 & 1231,0 \\
\hline 1 & 500 & 72,0 & $<0,9$ & 25,5 & 72,0 & $<0,9$ & 25,5 & $10: 11$ & 89,9 & 1231,5 \\
\hline 2 & 500 & 69,0 & $<0,9$ & 26,0 & 70,0 & $<0,9$ & 25,0 & $10: 22$ & 90,7 & 1232,0 \\
\hline 3 & 500 & 67,0 & $<0,9$ & 26,0 & 69,0 & $<0,9$ & 25,5 & $10: 33$ & 91,4 & 1232,5 \\
\hline 4 & 500 & 65,0 & 0,9040 & 26,5 & 67,9 & $<0,9$ & 25,5 & $10: 44$ & 91,9 & 1232,6 \\
\hline 5 & 500 & 62,0 & 0,9100 & 26,5 & 66,5 & $<0,9$ & 26,0 & $10: 52$ & 92,6 & 1232,9 \\
\hline 6 & 500 & 58,5 & 0,9165 & 26,5 & 65,0 & 0,920 & 26,0 & 11:08 & 93,3 & 1233,3 \\
\hline 7 & 500 & 55,0 & 0,9240 & 27,0 & 63,5 & 0,906 & 26,0 & $11: 21$ & 94,0 & 1233,9 \\
\hline 8 & 500 & 51,0 & 0,9320 & 26,5 & 61,5 & 0,910 & 26,0 & $11: 34$ & 94,7 & 1234,0 \\
\hline 9 & 500 & 46,0 & 0,9410 & 27,0 & 60,0 & 0,913 & 26,0 & 11:49 & 95,3 & 1234,5 \\
\hline 10 & 500 & 41,0 & 0,9500 & 27,0 & 58,0 & 0,917 & 26,0 & $12: 03$ & 95,9 & 1234,8 \\
\hline 11 & 500 & 36,0 & 0,9590 & 27,0 & 56,0 & 0,922 & 26,5 & $12: 20$ & 96,6 & 1235,0 \\
\hline 12 & 500 & 30,0 & 0,9660 & 27,0 & 53,5 & 0,927 & 26,5 & $12: 39$ & 97,3 & 1235,5 \\
\hline 13 & 500 & 23,0 & 0,9720 & 27,0 & 51,5 & 0,931 & 27,0 & $12: 50$ & 97,6 & 1235,9 \\
\hline 14 & 500 & 18,0 & 0,9780 & 27,0 & 49,5 & 0,936 & 27,0 & 13:06 & 98,0 & 1236,4 \\
\hline 15 & 500 & 14,0 & 0,9820 & 27,5 & 48,0 & 0,939 & 27,5 & $13: 23$ & 98,2 & 1236,7 \\
\hline
\end{tabular}


Tabla 4. Data recolectada en el control de cada corrida en el destilador Batch de 30litros.

\begin{tabular}{|c|c|c|c|c|c|c|c|c|c|c|}
\hline \multicolumn{4}{|c|}{$\begin{array}{l}\text { Fecha:25.04.06 } \\
\text { Primera Gota: 14:54 PM }\end{array}$} & \multicolumn{3}{|c|}{$\begin{array}{l}\text { Hora de inicio: 13:36 PM } \\
\text { T. Vapor }=87,2 \circ \mathrm{C}\end{array}$} & \multicolumn{4}{|c|}{$\begin{array}{l}\text { Volumen de Mosto: } 25 \mathrm{~L} \\
\text { Varcador de Energía: } 1238,9 \mathrm{Kw}\end{array}$} \\
\hline $\begin{array}{l}\text { № } \\
\text { Corte }\end{array}$ & $\begin{array}{l}\text { Volumen } \\
\mathrm{ml}\end{array}$ & $\begin{array}{c}\text { OG } \\
\text { muestra }\end{array}$ & $\begin{array}{l}\rho, \mathrm{gr} / \mathrm{ml} \\
\text { muestra }\end{array}$ & $\begin{array}{c}\text { To } \mathrm{C} \\
\text { muestra }\end{array}$ & $\begin{array}{c}\circ \mathrm{GL} \\
\text { mezcla }\end{array}$ & $\begin{array}{c}\rho, \mathbf{g r} / \mathrm{ml} \\
\text { mezcla }\end{array}$ & $\begin{array}{c}\text { To C } \\
\text { mezcla }\end{array}$ & Tiempo & $\begin{array}{l}\text { To C } \\
\text { Vapor }\end{array}$ & $\begin{array}{c}\text { Energía } \\
\mathrm{Kw}\end{array}$ \\
\hline Cabeza & 188 & 71,5 & $<0,9$ & 26,5 & 71,5 & $<0,9$ & 26,5 & $15: 00$ & 89,1 & 1239,1 \\
\hline 1 & 500 & 72,0 & $<0,9$ & 27,5 & 72,0 & $<0,9$ & 27,5 & $15: 10$ & 90,0 & 1239,3 \\
\hline 2 & 500 & 69,5 & $<0,9$ & 28,0 & 70,5 & $<0,9$ & 27,5 & $15: 20$ & 90,8 & 1239,6 \\
\hline 3 & 500 & 69,0 & $<0,9$ & 28,0 & 69,5 & $<0,9$ & 27,5 & $15: 31$ & 91,4 & 1239,9 \\
\hline 4 & 500 & 64,5 & 0,9040 & 27,5 & 68,5 & $<0,9$ & 27,5 & $15: 42$ & 92,1 & 1240,2 \\
\hline 5 & 500 & 61,0 & 0,9110 & 27,5 & 67,0 & $<0,9$ & 27,5 & $15: 54$ & 92,7 & 1240,5 \\
\hline 6 & 500 & 58,0 & 0,9180 & 28,0 & 65,0 & 0,9225 & 27,0 & $16: 06$ & 93,4 & 1240,9 \\
\hline 7 & 500 & 54,0 & 0,9260 & 28,0 & 63,5 & 0,9060 & 27,0 & $16: 18$ & 94,2 & 1241,2 \\
\hline 8 & 500 & 50,0 & 0,9350 & 28,0 & 62,0 & 0,9110 & 27,0 & $16: 31$ & 94,8 & 1241,6 \\
\hline 9 & 500 & 45,0 & 0,9440 & 27,0 & 59,5 & 0,9145 & 27,0 & $16: 45$ & 95,5 & 1241,9 \\
\hline 10 & 500 & 40,0 & 0,9530 & 27,0 & 57,0 & 0,9190 & 27,0 & 17:00 & 96,3 & 1242,3 \\
\hline 11 & 500 & 34,0 & 0,9610 & 27,5 & 55,5 & 0,9240 & 27,0 & $17: 15$ & 96,9 & 1242,7 \\
\hline 12 & 500 & 26,0 & 0,9685 & 27,5 & 53,0 & 0,9270 & 27,0 & $17: 30$ & 97,4 & 1243,2 \\
\hline 13 & 500 & 20,0 & 0,9750 & 27,0 & 50,0 & 0,9330 & 28,0 & $17: 46$ & 97,8 & 1243,6 \\
\hline 14 & 500 & 16,0 & 0,9800 & 27,0 & 48,0 & 0,9380 & 28,0 & 18:03 & 98,2 & 1244,0 \\
\hline 15 & 500 & 10,5 & 0,9860 & 27,05 & 46,0 & 0,9410 & 28,0 & $18: 20$ & 98,3 & 1244,4 \\
\hline
\end{tabular}

Una vez retirada la "cabeza", se sigue destilando y colectando los cortes que configuran el "cuerpo" y que se definen automáticamente en función al grado alcohólico de la mezcla. En el caso del proceso propuesto se trabaja en el rango de $46^{\circ}$ $48^{\circ} \mathrm{GL}$. El "cuerpo" es la porción del destilado que se transformará mediante un proceso de maduración de por lo menos 3 meses.

\section{Proceso de elaboración de aguardiente denominado Pisco}

La Fig.11 muestra el proceso de elaboración de aguardiente de uvas propuesto por los investigadores y que luego de más de 10 corridas completas se demostró que los rendimientos que se obtienen son mayores a los estándares actuales y con calidad garantizada del producto final.

\section{Empresa spin-off de Pisco UNI}

El producto resultante del proceso propuesto, muestra excelentes características organolépticas con referencia al mercado.
Por ejemplo a pesar de utilizar un tipo de uva que no es considerada aromática; como es el caso de la uva quebranta; se obtiene un pisco aromático, dulce, suave y terso que se puede tomar solo o combinado.

La calidad del aguardiente elaborado mediante el proceso propuesto fue acreditada por el CITEvid y por una diversidad de usuarios nacionales e internacionales a quien se le hizo catar.

El equipo de investigación procedió a formular la creación de una empresa spin-off de Pisco-UNI como resultado de la investigación aplicada y la innovación tecnológica realizada en la FIQT-UNI, ayudando de esta manera a trasferir el conocimiento y la investigación científica al mundo empresarial (principalmente a la industria artesanal) buscando su aplicación directa en los procesos [4]. 


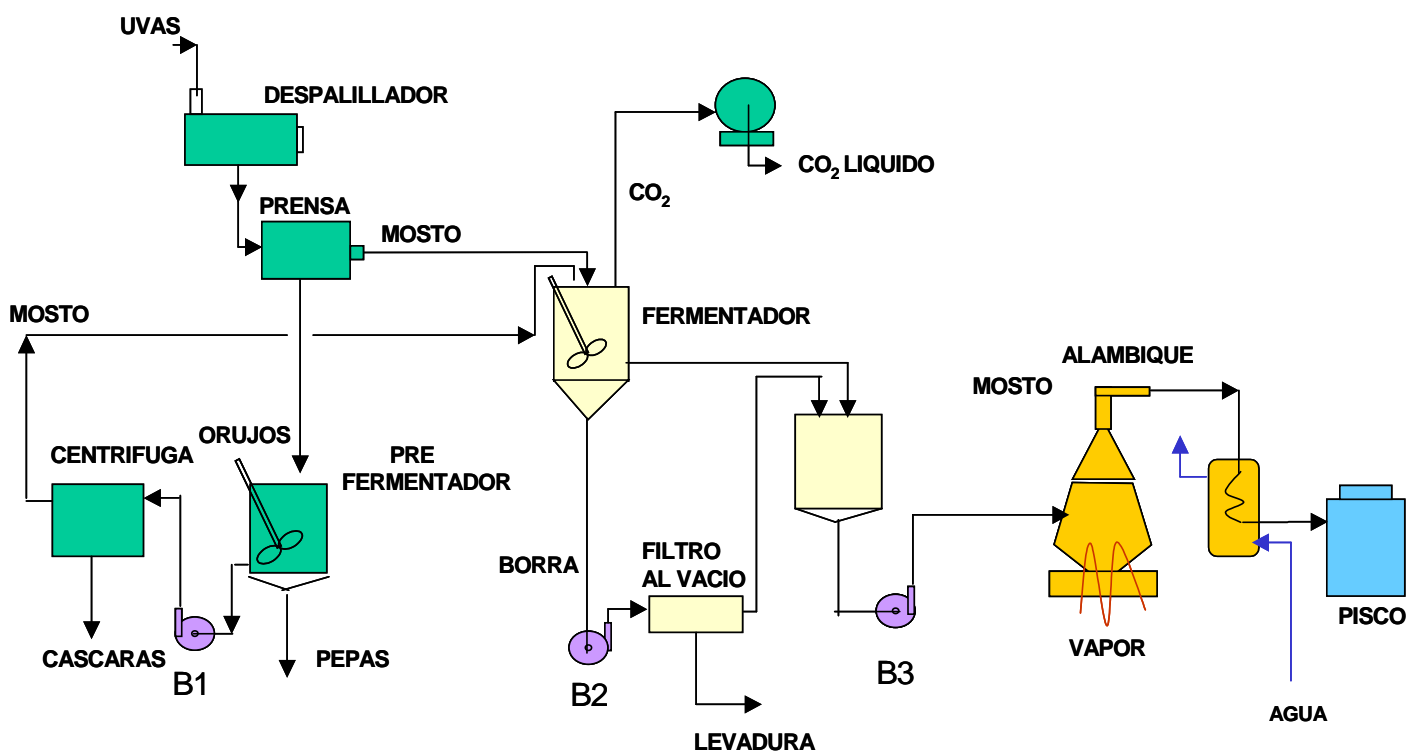

Fig. 11 Proceso definitivo propuesto por los investigadores para la elaboración de aguardiente de uvas denominado Pisco.

Se ofreció el pisco envasado en botellas debidamente selladas, con contratapas y etiquetadas con marca Pisco UNI, tal como se muestra en la Fig. 12.

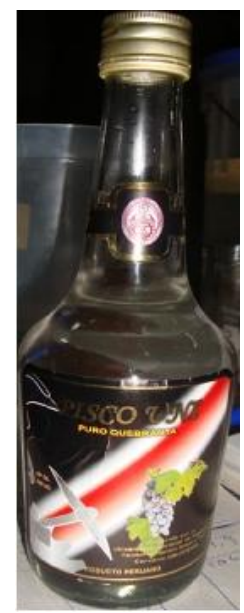

Fig. 12 Botella de Pisco-UNI con aguardiente de uva elaborado mediante el proceso propuesto por los investigadores.

\section{CONCLUSIONES}

Con las experiencias expuestas en el presente trabajo, se ha obtenido, un proceso para la elaboración de aguardiente de uva denominado
Pisco con especificaciones técnicas definidas y debidamente controladas.

A partir de los resultados obtenidos en investigaciones previas; dentro del marco del proyecto; principalmente de la Etapa escala Banco- Reactor de 45 litros, se diseñó como hipótesis un proceso preliminar para la elaboración de aguardiente de uvas denominado Pisco. A partir de éstas hipótesis se diseñaron y construyeron equipos a nivel de planta prototipo, principalmente un Reactor-Fermentador de 600 litros y se acondicionó un destilador batch debidamente implementado.

Se realizaron múltiples pruebas, principalmente en las etapas críticas de la fermentación del mosto de uvas y la posterior destilación del mosto fermentado. Se registró abundante data, que servirá para el diseño de una potencial planta industrial

Se incluyeron etapas innovadoras dentro del proceso convencional con resultados positivos de rendimiento y calidad, tales como la etapa de pre fermentación del "orujo", el control de la temperatura en la fermentación, la eliminación del "estrés" de la levadura y recuperación de la "borra" o lodo de levadura. 
Las anteriores innovaciones se tradujeron en una reducción dramática del consumo de la materia prima por unidad de producto sin menoscabo de la calidad la cual incluso mejoró. Se redujo el consumo estándar del mercado de 6-8 kilos de uva por litro de pisco producido a por debajo de los 5.0 kilos de uva por litro de pisco.

El proyecto, consideró la participación masiva de estudiantes investigadores a quienes se les orientó y entrenó en los procedimientos de la investigación aplicada con resultados óptimos.

Se determinó el potencial de los subproductos del proceso, tales, como el "escobajo" o ramas de los racimos, las cáscaras y las pepas de la uva original la cáscara. Asimismo la "borra" o lodo de levadura, el anhídrido carbónico producido masivamente en la fermentación, la "cabeza“" y la "cola" o vinaza en la destilación.

La Fig. 13, muestra la gran cantidad de productos que se pueden obtener a partir de los subproductos generados en el proceso propuesto por los investigadores, lo que serán consideran puestos en consideración en investigaciones futuras.

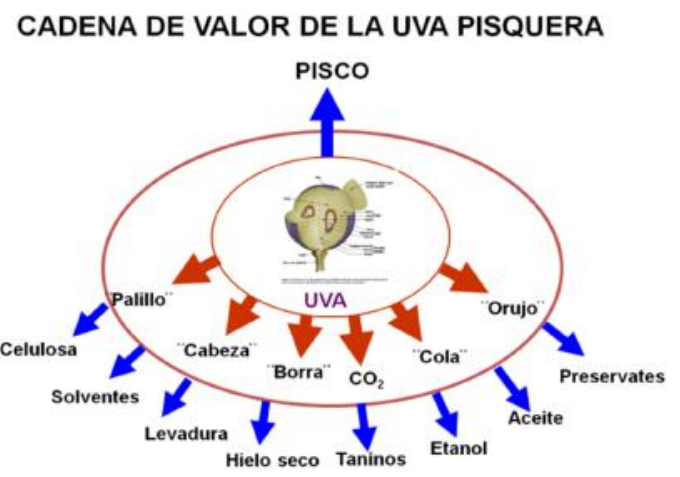

Fig. 13 Cadena de valor de la uva.

\section{AGRADECIMIENTOS}

A las autoridades de la FIQT, que confiaron en el equipo investigador durante todas las etapas del proyecto.

A docentes y alumnos investigadores que se comprometieron con el proyecto en todas sus etapas desde el año 2004, cuando se inició a escala laboratorio, como proyecto "Elaboración y especificaciones técnicas del pisco peruano".

Al Eco. Manuel Morón; Director Ejecutivo del CITEvid, promotor junto a los investigadores de la UNI en la suscripción de un convenio interinstitucional entre la UNI y el CITEvid. Asimismo, por su permanente apoyo a la continuación del proyecto.

Al Dr. Abad Flores Paucarima y su equipo de colaboradores en microbiología de la UNMSM, quienes enriquecieron la investigación biotecnológica a través de sus valiosos conocimientos y experiencia en la rama.

Al Ing. Emerson Collado Domínguez, que como Director del Instituto General de Investigación de la UNI (IGI-UNI), apoyó financieramente el proyecto en sus fases de escala Banco y escala Reactor - Fermentador de 600 litros y desde los inicios del proceso para la obtención de la Patente de Invención en INDECOPI.

Al Dr. Juan Rodríguez Rodríguez, actual Director del Instituto General de Investigación de la UNI (IGI-UNI), que continuó con el apoyo al proyecto en la gestión de la obtención de la Patente de Invención, hasta su culminación.

\section{REFERENCIAS}

1. Centro de Innovación Tecnológica Vitivinícola (CITEVID), "Programa de las Naciones Unidas para el Desarrollo (PNUD), "La Uva y el Pisco: Potencialidades productivas". Marzo del 2004.

2. Togores, J. H., "Tratado de Enología" Ediciones Mundi-Prensa, Madrid (España).

3. Flanzy, C., "Enología: Fundamentos Científicos y Tecnológicos", AMV Ediciones, Mundi Prensa (España) 20004.

4. Michael J. C. Martin, "Managing Innovation and Entrepreneurship in Technology-Based Firms”. John Wiley \& Sons, Sep 16, 1994.

5. Perry, R. H., Chilton, C. H., "Chemical Engineers' Handbook" Fifth Edition, Mc Graw-ill Kogakusha Ltd., 1973.

6. Schweitzer, P. A., Editor in Chief "Handbook of Separation Techniques for 
Chemical Engineers", Mc Graw-Hill Book Company, 1979.

7. Oldshue, J. Y., "Fluid Mixing Technology", Mc Graw-Hill Publications Co. New York, N.Y. 1983.
8. Uhl, V. W., Gray, J. B., "Mixing Theory and Practice", Vol. I (1966), Vol. II (1967), Academic Press Inc (London) Ltd. Mc GrawHill Publications Co. New York, N.Y. 1983.

Correspondencia:mario45@gmail.com 